\title{
Web-review: PET/CT imaging
}

\author{
IK Indrajit \\ Department of Radiodiagnosis and Imaging, Army Hospital (Research and Referral), Delhi Cantt - 110 010, India
}

Correspondence: Dr. IK Indrajit, Department of Radiodiagnosis and Imaging, Army Hospital (Research and Referral), Delhi Cantt - 110 010, India. E-mail: inji63@gmail.com

A few useful websites dealing with PET and PET/CT imaging, that are available at the moment on the Internet, are reviewed below:

1. Normal and benign pathological findings in 18 FDGPET and PET/CT: an interactive Web-based image atlas is available currently from http://www.jpnm. org/petctatlas.html or alternatively at http://www.med. harvard.edu/JPNM/chetan/. This educative and useful atlas designed and authored by CD Rajadyaksha, JA Parker, L Barbaras, and VH Gerbaudo is a result of a joint program in nuclear medicine based in Harvard Medical School. The descriptive sections commence with the basis of 18F-FDG and basis of PET/CT. It has illustrative material on normal, benign, and pathological variants, as well as common pitfalls and artifacts. A user friendly 'Case List' is available from http://www.med. harvard.edu/JPNM/chetan/caselist/caselist.html.

2. The Society of Nuclear Medicine has its official website at http://interactive.snm.org/. Sourced from Reston, Virginia, the website offers a range of educative features on PET and PET/CT. While some of the material is free, useful sections like 'Online Lectures' and 'Clinical Papers' on PET/CT are available after a mandatory registration. A useful link to 'Procedure Guidelines' for various regions and studies is available at http:// interactive.snm.org/index.cfm?PageID=772.

3. PET/CT education is an educative portal available from the PET Center, University of Southern California, Los Angeles, California. Sourced from https://petcenter. hsc.usc.edu/petcteducation/index.jsp, the website is created, designed, and administered by Daniel Cham, Gill Sunmeet, and Peter Conti. This website has two illustrative educational sections, the 'Atlas' and the 'Education' sections, covering a variety of cancerous lesions of the breast, cervix, colon and rectum, lung, oral cavity, and ovary. Besides these, PET/CT findings of malignancies like leukemia, lymphoma, melanoma, and multiple myeloma are also illustratively analyzed.

4. Positron emission tomography (PET/CT) at http:// www.nucmedinfo.com/Pages/petbase.html has a well- compiled list of materials featuring guidelines, teaching materials, atlas, readings, CME, and useful links on PET/CT. Designed by Hojjat Ahmadzadehfar, from the Department of Nuclear Medicine, University of Bonn, there are useful links to bone scintigraphy, breast cancer, cardiac imaging, scintigraphy, nuclear physics, oncology, radionuclide therapy, radiopharmacy, and tumor imaging.

5. PET/CT Basics is a self-tutorial for radiologists for learning the basics of PET/CT. It is authored by Rodney Bowman, Stewart Edmunds, Dominique Caovan et al. from the Department of Radiology, University of Virginia Health Sciences Center and is accessible at http://www.med-ed.virginia.edu/courses/rad/PETCT/ index.html. Similarly, The Place for PET: the Online Handbook for Referring Physicians and Imagers is sourced from Medscape at http://www.medscape. com/viewprogram/3825. Medscape is a comprehensive multispeciality resource, featuring CME modules, medical journal articles, Medline, etc. Authored by Chuong Bui and Barry Shulkin, this comprehensive piece on FDG-PET, highlights a range of issues, covering normal FDG distribution, clinical applications, and the 'expanding applications for PET scanning.'

6. IU PET is the official web portal featuring PET/ CT from Indiana University Radiology Associates available at http://www.indyrad.iupui.edu/radweb/ ResearchandImagingSciences/IUPET/tabid/242/Default. aspx. The website offers basic educational material in various sections comprising 'Overview of PET Applications,' 'FDG-PET and Lung Cancer Staging,' 'PET/CT Imaging,' and 'Patient Preparation.'

7. Diversified Pharmacy from the Eastern part of the United States, offers educational material online on PET/CT at http://www.dpgonline.com/cmes.php, as a part of their ongoing CME efforts. The material on PET/CT has been divided into cardiology, neurology, and oncology segments, each with common entities encountered in clinical practice. To illustrate, the section in cardiology deals with PET and PET/CT imaging in 
myocardial infarction, while neurology has FDG-PET brain imaging for differentiating Alzheimer's disease and other dementias, and oncology encompasses material on PET and PET/CT for radiation therapy planning and PET/CT cancer scanning.

8. PET/CT from Trident Molecular Imaging, Georgia, US is a dedicated and educational PET/CT portal at http://www.tridentpet.com/default.aspx. The material offered includes 'Clinical Applications,' 'Cases,' and 'Links.' Besides there is a separate webpage for archived newsletters, offered by this centre, titled 'The Stage,' which is sourced from http://www.tridentpet.com/ default.aspx?PID=2958. It illustratively covers various malignancies featured monthly from September 2004 (Solitary Pulmonary Nodule) to April 2007 (Pancreatic Cancer). Additionally, a set of PET/CT clinical algorithms on breast cancer, colorectal cancer, lung cancer, and lymphoma is available at http://www.tridentpet.com/ default.aspx?PID=2665.

9. Radiology Rounds is a regular newsletter for referring physicians, available from the Department of Radiology, Massachusetts General Hospital at http://www. mghradrounds.org/. Among the archived newsletters, there are two brief and comprehensive educational materials dealing with practical issues in PET/CT. The May 2004 issue focuses on 'PET/CT for Tumor Imaging' and is authored by Janet Miller and Susanna Lee, while the May 2007 issue authored by Janet Miller and Raul Uppot concentrates on an important issue in clinical practice, titled 'When to Select PET/CT vs Stand-Alone PET.'

10. A comprehensive 'Education Collections' of notable citations in PET/CT is available from Radiological Society of North America at http://www.rsna.org/ Education/archive/PET.cfm. A set of PET/CT cases is available from

a. Interactive PET/CT Case Of the Month from Guy's King's and St Thomas' (GKT) School of Medicine,
London is available at http://www-pet.umds.ac.uk/pic/ index.php?option=com_contentandtask=sectionandid $=9$ and Itemid $=40$

b. Westchester Medical Center, New York, titled University PET CT is available at http://www. universitypetct.com/samples/.

c. Franklin Square Hospital Center, Baltimore, Maryland at http://www.petcases.com/body.cfm?id=953andSubD omain=true

d. PETNET Solutions at http://www.petscaninfo.com/ zportal/portals/phys/clinical/case_month

\section{End piece}

'Case in Point' is a special educational section of the American College of Radiology available at http://caseinpoint.acr. org/index.htm. This website offers an interactive quiz compendium of cases in clinical practice. The editors of this user-friendly and frequently updated portal are Rona Woldenberg and Daniel M. Walz. The material is offered in two sections: 'Case in Point-Calendar' and 'Case in Point-Archive.' Additionally, one can periodically receive an e-mail alert for new cases displayed at this portal

Radiology Education.com is a 'digital library of radiology education resources,' created by Michael P. D'Alessandro. It is available at http://www.radiologyeducation.com/. It is a web portal providing 'a starting point for entry into radiology places of enlightenment, entertainment, and education on the Internet.' Available at this site are numerous links to quality educational portals across the net. They include radiology textbooks, radiology teaching files, CME gateways, materials for handheld computers, and multimedia items for podcasts. Besides, there are separate sections for residents as well as for medical students. Available at the end of the exhaustive webpage, are constructive links to tools that manage digital images, edit DICOM images, as well as assist in creation of Webbased teaching files.

Source of Support: Nil, Conflict of Interest: None declared. 